\title{
Stochastic Comparisons of Extreme Order Statistics in the Heterogeneous Exponentiated Scale Model
}

\author{
Esmaeil Bashkar \\ Department of Statistics, Yazd University, Yazd, 89175-741, Iran \\ e.bashkar@stu.yazd.ac.ir \\ Hamzeh Torabi \\ Department of Statistics, Yazd University, Yazd, 89175-741, Iran \\ htorabi@yazd.ac.ir \\ Rasool Roozegar \\ Department of Statistics, Yazd University, Yazd, 89175-741, Iran \\ rroozegar@yazd.ac.ir
}

Received 19 August 2016

Accepted 14 November 2016

\begin{abstract}
The effect of heterogeneity on order statistics has attracted much attention in recent decades. In this paper, first, we discuss stochastic comparisons of extreme order statistics from independent heterogeneous exponentiated scale samples. These comparisons are made with respect to usual stochastic, reversed hazard rate and likelihood ratio orderings. Then, in the presence of the Archimedean copula or survival copula for the random variables, we obtain the usual stochastic order of the sample extremes. In addition, some examples and applications are illustrated.
\end{abstract}

Keywords: Archimedean copula; exponentiated scale model; majorization; parallel system; series system.

2000 Mathematics Subject Classification: 60E15, 60K10, 62G30

\section{Introduction}

Suppose order statistics arising from random variables $X_{1}, \ldots, X_{n}$ are denoted by $X_{1: n} \leq \ldots \leq X_{n: n}$. Then it is well-known that the $k$ th order statistic of a sample of size $n$ characterizes the lifetime of a $(n-k+1)$-out-of- $n$ system. Thus, the study of lifetimes of $k$-out-of- $n$ systems is equivalent to the study of the stochastic properties of order statistics. In particular, a 1-out-of- $n$ system corresponds to a parallel system and an $n$-out-of- $n$ system corresponds to a series system. In extreme value theory, the extreme large loss such as stock market crashing or extreme short arrival times of disaster events could be naturally described by extreme order statistics. Examples and theoretical developments of extreme order statistics in both areas could be found in [2] and [7]. Also, the sample extremes have nice applications in auction theory. For example, the maximum and minimum define the final 
price of the sealed-bid first-price auction (FPA) and the first-price procurement auction. One may refer to [29], [28] and [19] for comprehensive expositions of auction theory, and we refer readers to [23] and [8] for applications of order statistics in auction theory. Besides, order statistics play a role in statistical inference, operation research, economics and many other applied probability fields. There are considerable studies on order statistics during the past several decades and a large number of which are on stochastic comparisons of order statistics from heterogeneous and homogeneous samples. Due to the complexity of the distribution theory, most existing research assumes the mutual independence among concerned random variables. For comprehensive references one may refer to [5], [15] and [3].

A random variable $X$ belongs to the scale family of distributions if $X \sim F(\lambda x)$, where $\lambda>0$ and $F$ is an absolutely continuous distribution function. Most of the parametric families of practical importance have a scale parameter, such as exponential, Weibull, gamma, and Pareto, etc. The scale model, also known in the literature as the proportional random variables model, is important in various fields of probability and statistics, see for example [32] and [10], among others. The scale model is termed as accelerated life models in the context of life testing because the scale parameter acts to control the rate at which time passes. There is an extensive literature on stochastic orderings when the observations follow from the exponential distribution with different scale parameters, see for instance, [32], [33], [6], [12], [17], [15] and the references therein. A natural way to extend these works is considering the scale model since it includes the exponential distribution, among others. Firstly, Khaledi and Kochar [11] studied conditions under which series and parallel systems consisting of components with lifetimes from the scale family of distributions are ordered in the hazard rate and the reverse hazard rate orderings, respectively. Then, Kochar and Torrado [16] have compared the magnitudes of two largest order statistics from the scale model when one set of scale parameters majorizes the other one. Li et al. [25] studied order statistics from random variables following the scale model. In the presence of the Archimedean copula or survival copula for the random variables, they obtained the usual stochastic order of the sample extremes.

Consider a distribution function $F$, and let $\alpha>0$. We know that $G(x)=(F(x))^{\alpha}$ is also a distribution function and is known as exponentiated distribution. It is also known as proportional reversed hazard rate model (PRHRM). A flexible model which belongs to the exponentiation family, is the exponentiated Weibull (EW) distribution proposed by Mudholkar and Srivastava [30]. The EW distribution is quite adequate for modeling non-monotone failure rates, including the bathtub shaped hazard rate, which are quite common in reliability and biological studies. Fang and Zhang [9] and Kundu and Chowdhury [20] stochastically compared two parallel systems each having heterogeneous EW components. Recently, a sub-model of the EW distribution, called the generalized exponential (GE) distribution, has been discussed extensively. Balakrishnan et al. [1] examined the problem of the stochastic comparison of series and parallel systems with heterogeneous GE components. The purpose of this paper is to study the magnitude of extreme order statistics from general distributions. More specifically, first, we discuss stochastic comparisons of extreme order statistics from independent heterogeneous exponentiated scale samples. These comparisons are made with respect to usual stochastic, reversed hazard rate and likelihood ratio orderings. Then, in the presence of the Archimedean copula or survival copula for the random variables, we obtain the usual stochastic order of the sample extremes. Some examples and applications are highlighted as well.

Recall that random variable $X$ belongs to the exponentiated scale family of distributions if $X \sim$ $H(x)=[F(\lambda x)]^{\alpha}$, where $\alpha, \lambda>0$ and $F$ is an absolutely continuous distribution function. We denote this family by $\operatorname{ES}(\alpha, \lambda)$. The exponentiated scale family is flexible enough to accommodate, 
in many cases, for both monotone as well as non-monotone hazard rates. In fact, $H(x)$ is quite different from $F(\lambda x)$ and need special investigation. For example, if $F(\lambda x)$ is exponential, then its corresponding PDF is monotone decreasing on the positive half of the real line. However, $H(x)=$ $\left(1-e^{-\lambda x}\right)^{\alpha}$ has a PDF, which is unimodal on $[0, \infty)$. Furthermore, while the exponential distribution has constant hazard rate $\lambda$, it can be shown that the generalized exponential $H$ has increasing hazard rate (IHR), if $\alpha>1$, constant hazard rate (CHR), if $\alpha=1$, and decreasing hazard rate (DHR) if $\alpha<1$. Let the corresponding density, hazard rate and reverse hazard rate functions of $F$ be $f, r$ and $\tilde{r}$ respectively. Recall that $F$ is said to be of

(i) Decreasing reversed hazard rate (denoted as DRHR) if $\tilde{r}(x)$ is decreasing;

(ii) Increasing hazard rate (denoted as IHR) if $r(x)$ is increasing;

(iii) Decreasing proportional reversed hazard rate (denoted as DPRHR) if $x \tilde{r}(x)$ is decreasing.

Notice that the reversed hazard rate function of $H$ is defined by

$$
\tilde{r}_{H}(x)=\alpha \lambda \tilde{r}(\lambda x)
$$

So that the RHRF of $H$ is proportional to the RHRF of $F$ with proportionality parameter $\alpha \lambda$.

Remarks

1 If in ES family, $\alpha=\lambda=1$, then $H(x)=F(x)$, which is also known as baseline distribution.

2 If $\lambda=1$, then $H(x)=(F(x))^{\alpha}$, which is also as exponentiated distribution.

3 If $\alpha=1$, then $H(x)=F(\lambda x)$, which is known as scale family.

The paper is organized as follows: Section 2 deals with different notions of stochastic orders, majorization and related orders. Some useful lemmas are given in this section. Section 3 contains the main result of the paper and its applications. Some conclusions are given in Section 4.

\section{The Basic Definitions and Some Prerequisites}

Throughout this paper, we use the notations $\mathbb{R}=(-\infty,+\infty), \mathbb{R}_{+}=[0,+\infty)$ and $\mathbb{R}_{++}=(0,+\infty)$, $\mathbb{D}_{+}=\left\{\left(x_{1}, x_{2}, \ldots, x_{n}\right): x_{1} \geq x_{2} \geq \cdots \geq x_{n}>0\right\}, \mathbb{E}_{+}=\left\{\left(x_{1}, x_{2}, \ldots, x_{n}\right): 0<x_{1} \leq x_{2} \leq \cdots \leq x_{n}\right\}$. The term increasing means non-decreasing and decreasing means non-increasing. For any differentiable function $f($.$) , we write the first derivative of f(t)$ with respect to $t$ by $f^{\prime}(t)$. We mention that all random variables are nonnegative throughout the paper.

In this section, we recall some notions of stochastic orders, majorization and related orders.

Let $X$ and $Y$ be two univariate random variables with distribution functions $F$ and $G$, density functions $f$ and $g$, the survival functions $\bar{F}=1-F$ and $\bar{G}=1-G$, hazard rate functions $r_{F}=$ $f / \bar{F}$ and $r_{G}=g / \bar{G}$, and reverse hazard rate functions $\tilde{r}_{F}=f / F$ and $\tilde{r}_{G}=g / G$, respectively. The following definition contains stochastic orders to compare the magnitudes of two random variables. For a comprehensive discussion on various stochastic orders, see [34] and [22].

Definition 2.1. Let $X$ and $Y$ be two nonnegative random variables on $\mathbb{R}_{++}$. The random variable $X$ is said to be smaller than $Y$ in the

(i) likelihood ratio order, denoted by $X \leq_{\operatorname{lr}} Y$, if $g(x) / f(x)$ is increasing in $x \in \mathbb{R}_{++}$,

(ii) hazard rate order, denoted by $X \leq_{\mathrm{hr}} Y$, if $r_{F}(x) \geq r_{G}(x)$ for all $x$,

(iii) reversed hazard rate order, denoted by $X \leq_{\mathrm{rh}} Y$, if $\tilde{r}_{F}(x) \leq \tilde{r}_{G}(x)$ for all $x$,

(iv) usual stochastic order, denoted by $X \leq_{\text {st }} Y$, if $\bar{F}(x) \leq \bar{G}(x)$ for all $x$. 
It is well known that the notion of majorization is quite useful in establishing various inequalities. Let us recall that the notation $x_{(1)} \leq x_{(2)} \leq \ldots \leq x_{(n)}$ is used to denote the increasing arrangement of the components of $\boldsymbol{x}=\left(x_{1}, \ldots, x_{n}\right)$.

Definition 2.2. The vector $x$ is said to be

(i) weakly submajorized by the vector $\boldsymbol{y}$ (denoted by $\boldsymbol{x} \preceq_{\mathrm{w}} \boldsymbol{y}$ ) if $\sum_{i=j}^{n} x_{(i)} \leq \sum_{i=j}^{n} y_{(i)}$ for all $j=1, \ldots, n$,

(ii) weakly supermajorized by the vector $\boldsymbol{y}$ (denoted by $\boldsymbol{x} \stackrel{\mathrm{w}}{\preceq} \boldsymbol{y}$ ) if $\sum_{i=1}^{j} x_{(i)} \geq \sum_{i=1}^{j} y_{(i)}$ for all $j=1, \ldots, n$,

(iii) majorized by the vector $\boldsymbol{y}$ (denoted by $\boldsymbol{x} \preceq \boldsymbol{m}$ ) if $\sum_{i=1}^{n} x_{i}=\sum_{i=1}^{n} y_{i}$ and $\sum_{i=1}^{j} x_{(i)} \geq \sum_{i=1}^{j} y_{(i)}$ for all $j=1, \ldots, n-1$.

Another interesting weaker order related to the majorization order is the $p$-larger order, introduced in [4].

Definition 2.3. A vector $\boldsymbol{x} \in \mathbb{R}_{+}^{n}$ is said to be $p$-larger than the vector $\boldsymbol{y} \in \mathbb{R}_{+}^{n}$ (denoted by $\boldsymbol{x} \succeq \boldsymbol{p}$ ) if

$$
\prod_{i=1}^{j} x_{(i)} \leq \prod_{i=1}^{j} y_{(i)}, \quad j=1, \ldots, n
$$

It is well-known that (cf. [13] and [18])

$$
\boldsymbol{x} \stackrel{\mathrm{p}}{\preceq} \Longleftarrow \boldsymbol{x} \stackrel{\mathrm{w}}{\preceq} \boldsymbol{y} \Longleftarrow \boldsymbol{x} \preceq \boldsymbol{m} \Longrightarrow \boldsymbol{x} \preceq{ }_{\mathrm{w}} \boldsymbol{y}, \quad \text { for } \boldsymbol{x}, \boldsymbol{y} \in \mathbb{R}_{+}^{n},
$$

and,

$$
\boldsymbol{x} \stackrel{\mathrm{p}}{\preceq} \boldsymbol{y} \Longleftrightarrow\left(\log \left(x_{1}\right), \ldots, \log \left(x_{n}\right)\right) \stackrel{\mathrm{w}}{\preceq}\left(\log \left(y_{1}\right), \ldots, \log \left(y_{n}\right)\right)
$$

Lemma 2.1 ( [26], Proposition 3.C.1). If $I \subset \mathbb{R}$ is an interval and $g: I \longrightarrow \mathbb{R}$ is convex, then $l(\boldsymbol{x})=\sum_{i=1}^{n} g\left(x_{i}\right)$ is Schur-convex on $I^{n}$. Consequently, $\boldsymbol{x} \preceq \boldsymbol{y}$ on $I^{n}$ implies $l(\boldsymbol{x}) \leq l(\boldsymbol{y})$.

Lemma 2.2 ( [26], Theorem 3.A.8). For a function $\phi$ on $\mathscr{A} \subset \mathbb{R}^{n}, \boldsymbol{x} \preceq_{\mathrm{w}}(\stackrel{\mathrm{w}}{\preceq}) \boldsymbol{y}$ implies $\boldsymbol{\phi}(\boldsymbol{x}) \leq \boldsymbol{\phi}(\boldsymbol{y})$ if and only if it is increasing (decreasing) and Schur-convex on $\mathscr{A}$.

A square matrix is said to be a permutation matrix if each row and each column has a single unit, and all other entries are zero. There exist $n$ ! such matrices of size $n \times n$, each of which is obtained by interchanging rows (or columns) of the identity matrix. An $n \times n$ matrix $P=\left(p_{i j}\right)$ is said to be doubly stochastic if $p_{i j} \geq 0$ for $i, j=1, \ldots, n, \sum_{i=1}^{n} p_{i j}=1, j=1, \ldots, n$ and $\sum_{j=1}^{n} p_{i j}=1$, $i=1, \ldots, n$. The T-transform matrix has the form $T_{\omega}=\omega I_{n}+(1-\omega) \Pi$, where $0 \leq \omega \leq 1, I_{n}$ is the $n \times n$ identity matrix and $\Pi$ is a permutation matrix that just interchanges two coordinates. In the following definition, various types of multivariate majorization are presented [26].

Definition 2.4. Let $A=\left\{a_{i j}\right\}$ and $B=\left\{b_{i j}\right\}$ be two $m \times n$ matrices such that $a_{1}^{R}, \ldots, a_{m}^{R}$, and $b_{1}^{R}, \ldots, b_{m}^{R}$ are the rows of $A$, and $B$, respectively.

(i) $A$ is said to chain majorize $B$ (denoted by $A \gg B$ ) if there exists a finite set of $n \times n T$ transform matrices $T_{\omega_{1}}, \ldots, T_{\omega_{k}}$ such that $B=A T_{\omega_{1}} \ldots T_{\omega_{k}}$. 
(ii) $A$ is said to majorize $B$ (denoted by $A>B$ ) if there exists an $n \times n$ doubly stochastic matrix $P$ such that $B=A P$.

(iii) $A$ is said to row majorize $B$ (denoted by $A>^{\text {row }} B$ ) if $a_{i}^{R} \succeq b_{i}^{R}$ for $i=1, \ldots, m$.

It is known that

$$
A \gg B \Longrightarrow A>B \Longrightarrow A>^{\text {row }} B
$$

Let

and

$$
\begin{aligned}
& S_{n}=\left\{(\boldsymbol{x}, \boldsymbol{y})=\left[\begin{array}{l}
x_{1}, \ldots, x_{n} \\
y_{1}, \ldots, y_{n}
\end{array}\right]: x_{i}>0, y_{i}>0, \text { and } \quad\left(x_{i}-x_{j}\right)\left(y_{i}-y_{j}\right) \leq 0,\right. \\
& i, j=1, \ldots, n\}
\end{aligned}
$$

$$
\begin{aligned}
& T_{n}=\left\{(\boldsymbol{x}, \boldsymbol{y})=\left[\begin{array}{l}
x_{1}, \ldots, x_{n} \\
y_{1}, \ldots, y_{n}
\end{array}\right]: x_{i} \geq 1, y_{i}>0, \text { and } \quad\left(x_{i}-x_{j}\right)\left(y_{i}-y_{j}\right) \leq 0,\right. \\
& i, j=1, \ldots, n\} .
\end{aligned}
$$

For a comprehensive treatment on this topic, see Chapter 15 of [26]. To prove the main results of this paper, we shall use the following theorems.

Theorem 2.1 ( [1]). A differentiable function $\varphi: \mathbb{R}_{+}^{4} \longrightarrow \mathbb{R}_{+}$satisfies

$$
\varphi(A) \geq \varphi(B) \text { for all } A, B \text { such that } A \in S_{2}\left(T_{2}\right), \text { and } A \gg B
$$

if and only if (iff)

(i) $\varphi(A)=\varphi(A \Pi)$ for all permutation matrices $\Pi$, and for all $A \in S_{2}\left(T_{2}\right)$; and

(ii) $\sum_{i=1}^{2}\left(a_{i k}-a_{i j}\right)\left(\varphi_{i k}(A)-\varphi_{i j}(A)\right) \geq 0$ for all $j, k=1,2$, and for all $A \in S_{2}\left(T_{2}\right)$, where $\varphi_{i j}(A)=\frac{\partial \varphi(A)}{\partial a_{i j}}$.

Theorem 2.2 ( [1]). Let $\Psi: \mathbb{R}_{+}^{2 n} \longrightarrow \mathbb{R}_{+}$be a differentiable function, and the function $v_{n}: \mathbb{R}_{+}^{2 n} \longrightarrow$ $\mathbb{R}_{+}$be defined as

$$
v_{n}(A)=\prod_{i=1}^{n} \Psi\left(a_{1 i}, a_{2 i}\right)
$$

Assume that $v_{2}$ satisfies (2.1). Then, for $A \in S_{n}\left(T_{n}\right)$, and $B=A T_{\omega}$, we have $v_{n}(A) \geq v_{n}(B)$.

Lemma 2.3 ( [1]). Let $\omega:(0, \infty) \times(0,1) \longrightarrow(-\infty, 0)$ be defined as

$$
\omega(\alpha, t)=\frac{\alpha^{t} \log (t)}{1-t^{\alpha}}
$$

Then,

(i) for each $0<t \leq 1, \omega(\alpha, t)$ is increasing with respect to $\alpha$; and

(ii) for each $\alpha \geq 0, \omega(\alpha, t)$ is decreasing with respect to $t$. 
Lemma 2.4 ( [13]). The function $\psi: \mathbb{R}_{+}^{n} \longrightarrow \mathbb{R}$ satisfies

$$
\boldsymbol{x} \stackrel{\mathrm{p}}{\preceq} \boldsymbol{y} \Longrightarrow \psi(\boldsymbol{x}) \leq \psi(\boldsymbol{y})
$$

if and only if;

(i) $\psi\left(e^{a_{1}}, \ldots, e^{a_{n}}\right)$ is Schur-convex in $\left(a_{1}, \ldots, a_{n}\right)$

(ii) $\psi\left(e^{a_{1}}, \ldots, e^{a_{n}}\right)$ is decreasing in $a_{i}$, for $i=1, \ldots, n$,

where $a_{i}=\log \left(x_{i}\right)$, for $i=1, \ldots, n$.

A real function $\phi$ is $n$-monotone on $(a, b) \subseteq(-\infty,+\infty)$ if $(-1)^{n-2} \phi^{(n-2)}$ is decreasing and convex in $(a, b)$ and $(-1)^{k} \phi^{(k)}(x) \geq 0$ for all $x \in(a, b), k=0,1, \ldots, n-2$, in which $\phi^{(i)}($.$) is the$ $i$ th derivative of $\phi($.$) . For a n$-monotone $(n \geq 2)$ function $\phi:[0,+\infty) \longrightarrow[0,1]$ with $\phi(0)=1$ and $\lim _{x \rightarrow+\infty} \phi(x)=0$, let $\psi=\phi,{ }^{-1}$ be the pseudo-inverse, then

$$
C_{\phi}\left(u_{1}, \ldots, u_{n}\right)=\phi\left(\psi\left(u_{1}\right)+\ldots+\psi\left(u_{n}\right)\right), \quad \text { for all } u_{i} \in[0,1], i=1, \ldots, n,
$$

is called an Archimedean copula with generator $\phi$. Archimedean copulas cover a wide range of dependence structures including the independence copula with generator $\phi(t)=e^{-t}$. For more on Archimedean copulas, readers may refer to [31] and [27].

At the end of this section, we recall a useful lemma, which plays an important role in the proofs of theorems in Section 4. Note that the two-dimensional case of lemma below had been proved in Theorem 4.4.2 of [31].

Lemma 2.5 ( [24] Lemma A.1). For two n-dimensional Archimedean copulas $C_{\phi_{1}}(\boldsymbol{u})$ and $C_{\phi_{2}}(\boldsymbol{u})$, if $\psi_{2} \circ \phi_{1}$ is super-additive, then $C_{\phi_{1}}(\boldsymbol{u}) \leq C_{\phi_{2}}(\boldsymbol{u})$ for all $u \in[0,1]^{n}$.

\section{Mutually Independent Variables}

Consider a system consisting of independent ES components with heterogeneity in both parameters. In this case, the specified parameters can be represented in a matrix form, and we can then examine the behavior of some of the aging functions of the lifetime of the system when the matrix of the parameters changes to another matrix in the sense of multivariate majorization. Theorem 3.1 and Theorem 3.2 discusses this problem for the case of parallel and series systems.

Theorem 3.1. Let $X_{1}, X_{2}$ be independent random variables with $X_{i} \sim \operatorname{ES}\left(\alpha_{i}, \lambda_{i}\right), i=1,2$. Further, let $X_{1}^{*}, X_{2}^{*}$ be another set of independent random variables with $X_{i}^{*} \sim \operatorname{ES}\left(\alpha_{i}^{*}, \lambda_{i}^{*}\right), i=1,2$ and $F$ is DRHR. Then, for $\left[\begin{array}{ll}\alpha_{1} & \alpha_{2} \\ \lambda_{1} & \lambda_{2}\end{array}\right] \in S_{2}$, we have

$$
\left[\begin{array}{ll}
\alpha_{1} & \alpha_{2} \\
\lambda_{1} & \lambda_{2}
\end{array}\right] \gg\left[\begin{array}{ll}
\alpha_{1}^{*} & \alpha_{2}^{*} \\
\lambda_{1}^{*} & \lambda_{2}^{*}
\end{array}\right] \Longrightarrow X_{2: 2} \geq_{\text {st }} X_{2: 2}^{*}
$$

Proof. The distribution function of $X_{2: 2}$ is

$$
F_{X_{2: 2}}(x)=\prod_{i=1}^{2}\left(F\left(\lambda_{i} x\right)\right)^{\alpha_{i}}, \quad x>0 .
$$

It is easy to see that, for fixed $x>0$, the function $F_{X_{2: 2}}(x)$ is permutation invariant in $\left(\alpha_{i}, \lambda_{i}\right)$, and so Condition (i) of Theorem 2.1 is satisfied. Next, we should show that Condition (ii) of Theorem 2.1 
is also satisfied. For fixed $x>0$, let us define the function $g$ as

$$
g(\boldsymbol{\alpha}, \boldsymbol{\lambda})=\left(\alpha_{1}-\alpha_{2}\right)\left(\frac{\partial F_{X_{2: 2}}(x)}{\partial \alpha_{1}}-\frac{\partial F_{X_{2: 2}}(x)}{\partial \alpha_{2}}\right)+\left(\lambda_{1}-\lambda_{2}\right)\left(\frac{\partial F_{X_{2: 2}}(x)}{\partial \lambda_{1}}-\frac{\partial F_{X_{2: 2}}(x)}{\partial \lambda_{2}}\right) .
$$

The partial derivatives of $F_{X_{2: 2}}(x)$ with respect to $\alpha_{i}$, and $\lambda_{i}$ are

$$
\frac{\partial F_{X_{2: 2}}(x)}{\partial \alpha_{i}}=F_{X_{2: 2}}(x) \log \left(F\left(\lambda_{i} x\right)\right)
$$

and

$$
\frac{\partial F_{X_{2: 2}}(x)}{\partial \lambda_{i}}=x F_{X_{2: 2}}(x) \alpha_{i} \tilde{r}\left(\lambda_{i} x\right)
$$

respectively. Now, upon substituting for these derivatives in (3.2), we get

$$
\begin{aligned}
g(\boldsymbol{\alpha}, \boldsymbol{\lambda})= & F_{X_{2: 2}}(x)\left(\alpha_{1}-\alpha_{2}\right)\left(\operatorname { l o g } \left(F\left(\lambda_{1} x\right)-\log \left(F\left(\lambda_{2} x\right)\right)+\right.\right. \\
& x F_{X_{2: 2}}(x)\left(\lambda_{1}-\lambda_{2}\right)\left(\alpha_{1} \tilde{r}\left(\lambda_{1} x\right)-\alpha_{2} \tilde{r}\left(\lambda_{2} x\right)\right) .
\end{aligned}
$$

The assumption $(\boldsymbol{\alpha}, \boldsymbol{\lambda}) \in S_{2}$ implies $\left(\alpha_{1}-\alpha_{2}\right)\left(\lambda_{1}-\lambda_{2}\right) \leq 0$. This result means that $\alpha_{1} \geq \alpha_{2}$ and $\lambda_{1} \leq \lambda_{2}$, or $\alpha_{1} \leq \alpha_{2}$ and $\lambda_{1} \geq \lambda_{2}$. We present the proof only for the case $\alpha_{1} \geq \alpha_{2}$ and $\lambda_{1} \leq \lambda_{2}$, because the proof for the other case is quite similar. Because $\log (F(\lambda x))$ is increasing with respect to $\lambda$, we have $\log \left(F\left(\lambda_{2} x\right)\right) \geq \log \left(F\left(\lambda_{1} x\right)\right)$. So, it follows that the first expression on the right hand side of (3.3) is non-positive. On the other hand, $\tilde{r}\left(\lambda_{2} x\right) \leq \tilde{r}\left(\lambda_{1} x\right)$, since $\tilde{r}(x)$ is decreasing by the assmption. By combining this observation with the assumption $\alpha_{1} \geq \alpha_{2}$, we see that the second term on the right hand side of (3.3) is also non-positive. Therefore, $g(\boldsymbol{\alpha}, \boldsymbol{\lambda}) \leq 0$, and this completes the proof of the theorem.

Theorem 3.2. Let $X_{1}, X_{2}\left(X_{1}^{*}, X_{2}^{*}\right)$ be independent random variables with $X_{i} \sim \operatorname{ES}\left(\alpha_{i}, \lambda_{i}\right)\left(X_{i}^{*} \sim\right.$ $\left.\operatorname{ES}\left(\alpha_{i}^{*}, \lambda_{i}^{*}\right)\right), i=1,2$ and let $F$ is IHR. Then, for $\left[\begin{array}{ll}\alpha_{1} & \alpha_{2} \\ \lambda_{1} & \lambda_{2}\end{array}\right] \in T_{2}$, we have

$$
\left[\begin{array}{ll}
\alpha_{1} & \alpha_{2} \\
\lambda_{1} & \lambda_{2}
\end{array}\right] \gg\left[\begin{array}{ll}
\alpha_{1}^{*} & \alpha_{2}^{*} \\
\lambda_{1}^{*} & \lambda_{2}^{*}
\end{array}\right] \Longrightarrow X_{1: 2}^{*} \geq_{\text {st }} X_{1: 2}
$$

Proof. The survival function of $X_{1: 2}$ is given by

$$
\bar{F}_{X_{1: 2}}(x)=\prod_{i=1}^{2}\left(1-\left(F\left(\lambda_{i} x\right)\right)^{\alpha_{i}}\right), \quad x>0 .
$$

For fixed $x>0$, the function $\bar{F}_{X_{1: 2}}(x)$ is permutation invariant in $\left(\alpha_{i}, \lambda_{i}\right)$, and so Condition (i) of Theorem 2.1 is satisfied. Next, we have to show that Condition (ii) of Theorem 2.1 also holds. For 
fixed $x>0$, consider the function $g$ as

$$
g(\boldsymbol{\alpha}, \boldsymbol{\lambda})=g_{1}(\boldsymbol{\alpha}, \boldsymbol{\lambda})+g_{2}(\boldsymbol{\alpha}, \boldsymbol{\lambda}),
$$

where

$$
g_{1}(\boldsymbol{\alpha}, \boldsymbol{\lambda})=\left(\alpha_{1}-\alpha_{2}\right)\left(\frac{\partial F_{X_{1: 2}}(x)}{\partial \alpha_{1}}-\frac{\partial F_{X_{1: 2}}(x)}{\partial \alpha_{2}}\right)
$$

and

$$
g_{2}(\boldsymbol{\alpha}, \boldsymbol{\lambda})=\left(\lambda_{1}-\lambda_{2}\right)\left(\frac{\partial F_{X_{1: 2}}(x)}{\partial \lambda_{1}}-\frac{\partial F_{X_{1: 2}}(x)}{\partial \lambda_{2}}\right) .
$$

The partial derivatives of $\bar{F}_{X_{1: 2}}(x)$ with respect to $\alpha_{i}$, and $\lambda_{i}$ are

$$
\frac{\partial \bar{F}_{X_{1: 2}}(x)}{\partial \alpha_{i}}=-\bar{F}_{X_{1: 2}}(x) \frac{\left(F\left(\lambda_{i} x\right)\right)^{\alpha_{i}} \ln \left(F\left(\lambda_{i} x\right)\right)}{1-\left(F\left(\lambda_{i} x\right)\right)^{\alpha_{i}}}
$$

and

$$
\frac{\partial \bar{F}_{X_{1: 2}}(x)}{\partial \lambda_{i}}=-x \alpha_{i} \bar{F}_{X_{1: 2}}(x) \frac{f\left(\lambda_{i} x\right)\left(F\left(\lambda_{i} x\right)\right)^{\alpha_{i}-1}}{1-\left(F\left(\lambda_{i} x\right)\right)^{\alpha_{i}}},
$$

respectively. Upon using (3.8) in (3.6), we get

$$
g_{1}(\boldsymbol{\alpha}, \boldsymbol{\lambda})=\bar{F}_{X_{1: 2}}(x)\left(\alpha_{1}-\alpha_{2}\right)\left(\omega\left(\alpha_{2}, F\left(\lambda_{2} x\right)\right)-\omega\left(\alpha_{1}, F\left(\lambda_{1} x\right)\right)\right) .
$$

where $\omega(\alpha, t)$ is as defined in Lemma 2.3. The assumption $(\boldsymbol{\alpha}, \lambda) \in T_{2}$ implies $\left(\alpha_{1}-\alpha_{2}\right)\left(\lambda_{1}-\lambda_{2}\right) \leq$ 0 . This result means that $\alpha_{1} \geq \alpha_{2} \geq 1$ and $\lambda_{1} \leq \lambda_{2}$, or $1 \leq \alpha_{1} \leq \alpha_{2}$ and $\lambda_{1} \geq \lambda_{2}$. We just express the proof for the case $\alpha_{1} \geq \alpha_{2} \geq 1$ and $\lambda_{1} \leq \lambda_{2}$, because the proof for the other case is very similar. From Lemma 2.3, it follows that $\omega(\alpha, F(\lambda x))$ is increasing with respect to $\alpha$ for fixed $\lambda$, and is decreasing with respect to $\lambda$ for fixed $\alpha$. Therefore, we can conclude that

$$
\omega\left(\alpha_{1}, F\left(\lambda_{1} x\right)\right) \geq \omega\left(\alpha_{2}, F\left(\lambda_{1} x\right)\right) \geq \omega\left(\alpha_{2}, F\left(\lambda_{2} x\right)\right),
$$

which in turn implies

$$
g_{1}(\boldsymbol{\alpha}, \boldsymbol{\lambda}) \leq 0 .
$$

On the other hand, upon using (3.9) in (3.7), we get

$$
\begin{aligned}
g_{2}(\boldsymbol{\alpha}, \boldsymbol{\lambda})= & x \bar{F}_{X_{1: 2}}(x)\left(\lambda_{1}-\lambda_{2}\right)\left(\alpha_{2} r\left(\lambda_{2} x\right) \frac{\left(F\left(\lambda_{2} x\right)\right)^{\alpha_{2}}}{1-\left(F\left(\lambda_{2} x\right)\right)^{\alpha_{2}}}-\right. \\
& \left.\alpha_{1} r\left(\lambda_{1} x\right) \frac{\left(F\left(\lambda_{1} x\right)\right)^{\alpha_{1}}}{1-\left(F\left(\lambda_{1} x\right)\right)^{\alpha_{1}}}\right) .
\end{aligned}
$$

Now, let us consider the function $h(\alpha)=\alpha \frac{(F(\lambda x))^{\alpha}}{1-(F(\lambda x))^{\alpha}}$. From Lemma 2.8 of [35], it follows that $h(\alpha)$ is decreasing with respect to $\alpha$ for fixed $\lambda$. The assumption $r(x)$ is increasing in $x$ implies that 
$r(x) \frac{F^{\alpha}(x)}{1-F^{\alpha}(x)}$ is increasing in $x$ for fixed $\alpha$. Hence,

$$
\begin{aligned}
& \alpha_{2} r\left(\lambda_{2} x\right) \frac{\left(F\left(\lambda_{2} x\right)\right)^{\alpha_{2}}}{1-\left(F\left(\lambda_{2} x\right)\right)^{\alpha_{2}}} \geq \alpha_{1} r\left(\lambda_{2} x\right) \frac{\left(F\left(\lambda_{2} x\right)\right)^{\alpha_{1}}}{1-\left(F\left(\lambda_{2} x\right)\right)^{\alpha_{1}}} \\
& \left.\geq \alpha_{1} r\left(\lambda_{1} x\right) \frac{\left(F\left(\lambda_{1} x\right)\right)^{\alpha_{1}}}{1-\left(F\left(\lambda_{1} x\right)\right)^{\alpha_{1}}}\right),
\end{aligned}
$$

and therefore

$$
g_{2}(\boldsymbol{\alpha}, \boldsymbol{\lambda}) \leq 0
$$

From (3.5), (3.10), and (3.11), Condition (ii) of Theorem 2.1 is satisfied, and this observation completes the proof of the theorem.

Next, we extend the special form of Theorem 3.1 and Theorem 3.2 to the case $n \geq 3$.

Theorem 3.3. Let $X_{1}, \ldots, X_{n}$ be a set of independent random variables with $X_{i} \sim \operatorname{ES}\left(\alpha_{i}, \lambda_{i}\right)$, $i=1, \ldots, n$. Further, let $X_{1}^{*}, \ldots, X_{n}^{*}$ be another set of independent random variables with $X_{i}^{*} \sim$ $\mathrm{ES}\left(\alpha_{i}^{*}, \lambda_{i}^{*}\right), i=1, \ldots, n$.

(i) If $F$ is DRHR, $\left[\begin{array}{lll}\alpha_{1} & \cdots & \alpha_{n} \\ \lambda_{1} & \cdots & \lambda_{n}\end{array}\right] \in S_{n}$ and

$$
\left[\begin{array}{lll}
\alpha_{1}^{*} & \cdots & \alpha_{n}^{*} \\
\lambda_{1}^{*} & \cdots & \lambda_{n}^{*}
\end{array}\right]=\left[\begin{array}{lll}
\alpha_{1} & \cdots & \alpha_{n} \\
\lambda_{1} & \cdots & \lambda_{n}
\end{array}\right] T_{\omega}
$$

then $X_{n: n} \geq_{\mathrm{st}} X_{n: n}^{*}$.

(ii) If $F$ is IHR, $\left[\begin{array}{lll}\alpha_{1} & \cdots & \alpha_{n} \\ \lambda_{1} & \cdots & \lambda_{n}\end{array}\right] \in T_{n}$ and

$$
\left[\begin{array}{lll}
\alpha_{1}^{*} & \cdots & \alpha_{n}^{*} \\
\lambda_{1}^{*} & \cdots & \lambda_{n}^{*}
\end{array}\right]=\left[\begin{array}{lll}
\alpha_{1} & \cdots & \alpha_{n} \\
\lambda_{1} & \cdots & \lambda_{n}
\end{array}\right] T_{\omega}
$$

then $X_{1: n}^{*} \geq_{\mathrm{st}} X_{1: n}$.

Proof.

(i) For fixed $x>0$, let $v_{n}(\boldsymbol{\alpha}, \boldsymbol{\lambda})=F_{X_{n: n}}(x)$, and $\Psi(\alpha, \lambda)=(F(\lambda x))^{\alpha}$. Then, we have $v_{n}(\boldsymbol{\alpha}, \boldsymbol{\lambda})=$ $\prod_{i=1}^{n} \Psi\left(\alpha_{i}, \lambda_{i}\right)$. As shown in Theorem 3.1, $v_{2}$ is satisfied in (2.1). Now, the desired result follows from Theorem 2.2.

(ii) The proof is similar to that of Part (i), and it is therefore omitted here for the sake of brevity.

As we know, the product of the two $T$-transform matrices with the same structures is a $T$ transform matrix. By using the induction method, one can easily see that the finite product of $T$ transform matrices with the same structures is also a $T$-transform matrix. Now, according to this discussion, we immediately obtain the following corollary from Theorem 3.3.

Corollary 3.1. Suppose $X_{i}$ and $X_{i}^{*}$ satisfy the assumptions of Theorem 3.3. 
(i) Assume that $\tilde{r}(x)$ is decreasing in $x,\left[\begin{array}{lll}\alpha_{1} & \cdots & \alpha_{n} \\ \lambda_{1} & \cdots & \lambda_{n}\end{array}\right] \in S_{n}$ and

$$
\left[\begin{array}{ccc}
\alpha_{1}^{*} & \cdots & \alpha_{n}^{*} \\
\lambda_{1}^{*} & \cdots & \lambda_{n}^{*}
\end{array}\right]=\left[\begin{array}{ccc}
\alpha_{1} & \cdots & \alpha_{n} \\
\lambda_{1} & \cdots & \lambda_{n}
\end{array}\right] T_{\omega_{1}} \cdots T_{\omega_{k}}
$$

where $T_{\omega_{i}}, i=1, \ldots, k$, have the same structures. Then, we have $X_{n: n} \geq_{s t} X_{n: n}^{*}$.

(ii) Suppose $r(x)$ is increasing in $x,\left[\begin{array}{lll}\alpha_{1} & \cdots & \alpha_{n} \\ \lambda_{1} & \cdots & \lambda_{n}\end{array}\right] \in T_{n}$ and

$$
\left[\begin{array}{ccc}
\alpha_{1}^{*} & \cdots & \alpha_{n}^{*} \\
\lambda_{1}^{*} & \cdots & \lambda_{n}^{*}
\end{array}\right]=\left[\begin{array}{lll}
\alpha_{1} & \cdots & \alpha_{n} \\
\lambda_{1} & \cdots & \lambda_{n}
\end{array}\right] T_{\omega_{1}} \cdots T_{\omega_{k}}
$$

where $T_{\omega_{i}}, i=1, \ldots, k$, have the same structures. Then, we have $X_{1: n}^{*} \geq_{s t} X_{1: n}$.

It is of interest to know whether the results of Corollary 3.1 may still hold if the matrices $T_{\omega_{i}}$, $i=1, \ldots, k$, have not the same structures. The following theorem gives an answer.

Theorem 3.4. Let $X_{1}, \ldots, X_{n}$ be a set of independent random variables with $X_{i} \sim \operatorname{ES}\left(\alpha_{i}, \lambda_{i}\right)$, $i=1, \ldots, n$. Further, let $X_{1}^{*}, \ldots, X_{n}^{*}$ be another set of independent random variables with $X_{i}^{*} \sim$ $\mathrm{ES}\left(\alpha_{i}^{*}, \lambda_{i}^{*}\right), i=1, \ldots, n$.

(i) Suppose that $F$ is DRHR, $\left[\begin{array}{lll}\alpha_{1} & \cdots & \alpha_{n} \\ \lambda_{1} & \cdots & \lambda_{n}\end{array}\right] \in S_{n}$,

$$
\left[\begin{array}{ccc}
\alpha_{1}^{*} & \cdots & \alpha_{n}^{*} \\
\lambda_{1}^{*} & \cdots & \lambda_{n}^{*}
\end{array}\right]=\left[\begin{array}{lll}
\alpha_{1} & \cdots & \alpha_{n} \\
\lambda_{1} & \cdots & \lambda_{n}
\end{array}\right] T_{\omega_{1}} \cdots T_{\omega_{i}} \in S_{n}
$$

for $i=1, \ldots, k-1, k \geq 2$ and

$$
\left[\begin{array}{lll}
\alpha_{1}^{*} & \cdots & \alpha_{n}^{*} \\
\lambda_{1}^{*} & \cdots & \lambda_{n}^{*}
\end{array}\right]=\left[\begin{array}{lll}
\alpha_{1} & \cdots & \alpha_{n} \\
\lambda_{1} & \cdots & \lambda_{n}
\end{array}\right] T_{\omega_{1}} \cdots T_{\omega_{k}}
$$

then $X_{n: n} \geq_{\mathrm{st}} X_{n: n}^{*}$

(ii) If $F$ is IHR, $\left[\begin{array}{lll}\alpha_{1} & \cdots & \alpha_{n} \\ \lambda_{1} & \cdots & \lambda_{n}\end{array}\right] \in T_{n}$,

$$
\left[\begin{array}{lll}
\alpha_{1}^{*} & \cdots & \alpha_{n}^{*} \\
\lambda_{1}^{*} & \cdots & \lambda_{n}^{*}
\end{array}\right]=\left[\begin{array}{lll}
\alpha_{1} & \cdots & \alpha_{n} \\
\lambda_{1} & \cdots & \lambda_{n}
\end{array}\right] T_{\omega_{1}} \cdots T_{\omega_{i}} \in S_{n}
$$

for $i=1, \ldots, k-1, k \geq 2$ and

$$
\left[\begin{array}{ccc}
\alpha_{1}^{*} & \cdots & \alpha_{n}^{*} \\
\lambda_{1}^{*} & \cdots & \lambda_{n}^{*}
\end{array}\right]=\left[\begin{array}{ccc}
\alpha_{1} & \cdots & \alpha_{n} \\
\lambda_{1} & \cdots & \lambda_{n}
\end{array}\right] T_{\omega_{1}} \cdots T_{\omega_{k}}
$$

then, we have $X_{1: n}^{*} \geq_{\text {st }} X_{1: n}$.

Proof. Set

$$
\left[\begin{array}{lll}
\alpha_{1}^{(j)} & \cdots & \alpha_{n}^{(j)} \\
\lambda_{1}^{(j)} & \cdots & \lambda_{n}^{(j)}
\end{array}\right]=\left[\begin{array}{lll}
\alpha_{1} \cdots & \alpha_{n} \\
\lambda_{1} & \cdots & \lambda_{n}
\end{array}\right] T_{\omega_{1}} \cdots T_{\omega_{j}}, \quad j=1, \ldots, k-1 .
$$

Let $Y_{1}^{(j)}, \ldots, Y_{n}^{(j)}, j=1, \ldots, k-1$, be the sets of independent random variables with $Y_{i}^{(j)} \sim$ $\mathrm{ES}\left(\alpha_{i}^{(j)}, \lambda_{i}^{(j)}\right), i=1, \ldots, n$, and $j=1, \ldots, k-1$. 
(i) From the assumption of the theorem, it follows that

$$
\left[\begin{array}{lll}
\alpha_{1}^{(j)} & \cdots & \alpha_{n}^{(j)} \\
\lambda_{1}^{(j)} & \cdots & \lambda_{n}^{(j)}
\end{array}\right] \in S_{n}
$$

for $j=1, \ldots, k-1$. Using these observations, and the result of Theorem 3.3, it follows that $X_{n: n} \geq_{s t} Y_{n: n}^{(1)} \geq_{s t} \cdots \geq_{s t} Y_{n: n}^{(k-1)} \geq_{s t} X_{n: n}^{*}$. This completes the proof of Part (i).

(ii) The proof is similar to that of Part (i), and is therefore omitted here.

In the following theorem, we compare parallel systems with independent heterogeneous ES components when one of the parameters is fixed, and the results are then developed with respect to the other parameter.

Theorem 3.5. For $i=1, \ldots, n$, let $X_{i}$ and $X_{i}^{*}$ be two sets of mutually independent random variables with $X_{i} \sim \operatorname{ES}\left(\alpha_{i}, \lambda\right)$ and $X_{i}^{*} \sim \operatorname{ES}\left(\alpha_{i}^{*}, \lambda\right)$. If $\sum_{i=1}^{n} \alpha_{i} \geq \sum_{i=1}^{n} \alpha_{i}^{*}$, then for any $\lambda>0$, we have $X_{n: n} \geq \operatorname{lr}$ $X_{n: n}^{*}$.

Proof. For $x>0$, the ratio of the density functions of $X_{n: n}$ and $X_{n: n}^{*}$ is

$$
\frac{f_{n}(x)}{g_{n}(x)}=\frac{\sum_{i=1}^{n} \alpha_{i}}{\sum_{i=1}^{n} \alpha_{i}^{*}}(F(\lambda x))^{\beta},
$$

where $\beta=\sum_{i=1}^{n} \alpha_{i}-\sum_{i=1}^{n} \alpha_{i}^{*}$. Because $\beta>0, \frac{f_{n}(x)}{g_{n}(x)}$ is increasing in $x$. This completes the proof of the required result.

Remark 3.1. It is worthwhile to note that $\left(\alpha_{1}, \ldots, \alpha_{n}\right) \succeq_{w}\left(\alpha_{1}^{*}, \ldots, \alpha_{n}^{*}\right)$ implies $\sum_{i=1}^{n} \alpha_{i} \geq \sum_{i=1}^{n} \alpha_{i}^{*}$. So, the condition $\sum_{i=1}^{n} \alpha_{i} \geq \sum_{i=1}^{n} \alpha_{i}^{*}$ in Theorem 3.5 is weaker than the weak submajorization order. Therefore, the result of Theorem 3.5 remains true under the weak submajorization order between shape parameters. In other words, we have the following result:

$$
\left(\alpha_{1}, \ldots, \alpha_{n}\right) \succeq_{w}\left(\alpha_{1}^{*}, \ldots, \alpha_{n}^{*}\right) \Longrightarrow X_{n: n} \geq_{l r} X_{n: n}^{*}
$$

also, it is easy to show that $\left(\alpha_{1}, \ldots, \alpha_{n}\right) \stackrel{\mathrm{W}}{\succeq}\left(\alpha_{1}^{*}, \ldots, \alpha_{n}^{*}\right)$ implies $\sum_{i=1}^{n} \alpha_{i} \leq \sum_{i=1}^{n} \alpha_{i}^{*}$. Then, according to Theorem 3.5, we have the following result:

$$
\left(\alpha_{1}, \ldots, \alpha_{n}\right) \stackrel{\mathrm{w}}{\succeq}\left(\alpha_{1}^{*}, \ldots, \alpha_{n}^{*}\right) \Longrightarrow X_{n: n} \leq_{l r} X_{n: n}^{*} .
$$

The following corollary, due to [36], provides some sufficient conditions for comparing the largest order statistics from two heterogeneous PRHR samples.

Corollary 3.2. Let $\left(X_{1}, X_{2}, \ldots, X_{n}\right)$ be a vector of independent random variables with $X_{i} \sim F^{\alpha_{i}}$ for $i=1, \ldots, n$. Let $\left(X_{1}^{*}, X_{2}^{*}, \ldots, X_{n}^{*}\right)$ be another vector of independent random variables with $X_{i}^{*} \sim F^{\alpha_{i}^{*}}$ for $i=1, \ldots, n$. Then,

$$
\left(\alpha_{1}, \ldots, \alpha_{n}\right) \stackrel{\mathrm{w}}{\succeq}\left(\alpha_{1}^{*}, \ldots, \alpha_{n}^{*}\right) \Longrightarrow X_{n: n} \leq_{\mathrm{rh}} X_{n: n}^{*} .
$$


Note that the result in Theorem 3.5 contains the case when $\lambda=1$, from which we have the following corollary for the PRHR model, which extends the above results of [36] in the context that the super-majorization in (3.14) is relaxed to $\sum_{i=1}^{n} \alpha_{i} \geq \sum_{i=1}^{n} \alpha_{i}^{*}$. Moreover, the reverse hazard rate order in (3.14) is also generalized to likelihood ratio order.

Corollary 3.3. Let $\left(X_{1}, X_{2}, \ldots, X_{n}\right)$ be a vector of independent random variables with $X_{i} \sim F^{\alpha_{i}}$ for $i=1, \ldots, n$. Let $\left(X_{1}^{*}, X_{2}^{*}, \ldots, X_{n}^{*}\right)$ be another vector of independent random variables with $X_{i}^{*} \sim F^{\alpha_{i}^{*}}$ for $i=1, \ldots, n$. Then,

$$
\sum_{i=1}^{n} \alpha_{i} \geq \sum_{i=1}^{n} \alpha_{i}^{*} \Longrightarrow X_{n: n} \geq_{l r} X_{n: n}^{*}
$$

In the next theorem, we compare parallel systems in the case when the two sets of scale parameters weakly majorize each other.

Theorem 3.6. Let $X_{1}, \ldots, X_{n}$ be a set of independent nonnegative random variables with $X_{i} \sim$ $\mathrm{ES}\left(\alpha, \lambda_{i}\right), i=1, \ldots, n$. Let $X_{1}^{*}, \ldots, X_{n}^{*}$ be another set of independent nonnegative random variables with $X_{i}^{*} \sim \operatorname{ES}\left(\alpha, \lambda_{i}^{*}\right), i=1, \ldots, n$. If $F$ is DPRHR and $x^{2} \tilde{r}^{\prime}(x)$ is increasing in $x$, then for any $\alpha>0$,

$$
\left(\lambda_{1}, \ldots, \lambda_{n}\right) \stackrel{\mathrm{w}}{\succeq}\left(\lambda_{1}^{*}, \ldots, \lambda_{n}^{*}\right) \Longrightarrow X_{n: n} \geq_{\mathrm{rh}} X_{n: n}^{*}
$$

Proof. Fix $x>0$. Then the reverse hazard rate of $X_{n: n}$ is

$$
\tilde{r}_{X_{n: n}}(x, \lambda)=\sum_{i=1}^{n} \alpha \lambda_{i} \tilde{r}\left(\lambda_{i} x\right)=\frac{\alpha}{x} \sum_{i=1}^{n} \varphi\left(\lambda_{i} x\right),
$$

where $\varphi(x)=x \tilde{r}(x), x \geq 0$. From Lemma 2.2 it suffices to show that, for each $x>0, \tilde{r}_{X_{n: n}}(x, \boldsymbol{\lambda})$ is Schur-convex and decreasing in $\lambda_{i}$ 's.

By the assumptions, $\varphi(x)$ is decreasing in $x$, then the reverse hazard rate function of $X_{n: n}$ is decreasing in each $\lambda_{i}$.

Now, from Lemma 2.1, the convexity of $\varphi(x)$ is needed to prove Schur-convexity of $\tilde{r}_{X_{n: n}}(x, \lambda)$.

Note that the assumption $x^{2} \tilde{r}^{\prime}(x)$ is increasing in $x$ is equivalent to $\tilde{r}(x)+x \tilde{r}^{\prime}(x)$ is increasing in $x$ since

$$
\left[x^{2} \tilde{r}^{\prime}(x)\right]^{\prime}=x\left(2 \tilde{r}^{\prime}(x)+x \tilde{r}^{\prime \prime}(x)\right)=x\left[\tilde{r}(x)+x \tilde{r}^{\prime}(x)\right]^{\prime},
$$

and $\tilde{r}(x)+x \tilde{r}^{\prime}(x)$ is increasing in $x$ is equivalent to $x \tilde{r}(x)$ is convex since

$$
[x \tilde{r}(x)]^{\prime}=\tilde{r}(x)+x \tilde{r}^{\prime}(x) .
$$

Hence, $\varphi(x)$ is convex. This completes the proof of theorem.

letting $\alpha=1$ in Theorem 3.6 leads to the following corollary, verified by [16].

Corollary 3.4. Let $X_{1}, \ldots, X_{n}$ be a set of independent nonnegative random variables with $X_{i} \sim$ $F\left(\lambda_{i} x\right), i=1, \ldots, n$, where $F$ is an absolutely continuous distribution function with density function f. Let $X_{1}^{*}, \ldots, X_{n}^{*}$ be another set of independent nonnegative random variables with $X_{i}^{*} \sim F\left(\lambda_{i}^{*} x\right)$, $i=1, \ldots, n$. If $x \tilde{r}(x)$ is decreasing in $x$ and $x^{2} \tilde{r}^{\prime}(x)$ is increasing in $x$, then,

$$
\left(\lambda_{1}, \ldots, \lambda_{n}\right) \stackrel{\mathrm{w}}{\succeq}\left(\lambda_{1}^{*}, \ldots, \lambda_{n}^{*}\right) \Longrightarrow X_{n: n} \geq_{r h} X_{n: n}^{*} .
$$


The following two theorems show that under certain conditions on parameters, there exists reversed hazard rate ordering between $X_{n: n}$ and $X_{n: n}^{*}$.

Theorem 3.7. For $i=1, \ldots, n$, let $X_{i}$ and $X_{i}^{*}$ be two sets of mutually independent random variables with $X_{i} \sim \mathrm{ES}\left(\alpha_{i}, \lambda_{i}\right)$ and $X_{i}^{*} \sim \mathrm{ES}\left(\alpha_{i}, \lambda_{i}^{*}\right)$. Further, suppose that $\left\{\boldsymbol{\alpha} \in \mathbb{E}_{+}, \boldsymbol{\lambda}, \boldsymbol{\lambda}^{*} \in \mathbb{D}_{+}\right\}(\{\boldsymbol{\alpha} \in$ $\left.\left.\mathbb{D}_{+}, \boldsymbol{\lambda}, \lambda^{*} \in \mathbb{E}_{+}\right\}\right)$and let $x \tilde{r}(x)$ be decreasing convex function of $x$. Then,

$$
\left(\lambda_{1}, \ldots, \lambda_{n}\right) \stackrel{\mathrm{w}}{\succeq}\left(\lambda_{1}^{*}, \ldots, \lambda_{n}^{*}\right) \Longrightarrow X_{n: n} \geq_{\mathrm{rh}} X_{n: n}^{*}
$$

Proof. Fix $x>0$. Then the reverse hazard rate of $X_{n: n}$ is

$$
\tilde{r}_{X_{n: n}}(x, \lambda)=\sum_{i=1}^{n} \alpha_{i} \lambda_{i} \tilde{r}\left(\lambda_{i} x\right)=\frac{1}{x} \sum_{i=1}^{n} \alpha_{i} \lambda_{i} x \tilde{r}\left(\lambda_{i} x\right)=\frac{1}{x} \sum_{i=1}^{n} \alpha_{i} g\left(\lambda_{i} x\right),
$$

where $g(x)=x \tilde{r}(x), x \geq 0$. From Lemma 2.2 it suffices to show that, for each $x>0, \tilde{r}_{X_{n: n}}(x, \boldsymbol{\lambda})$ is Schur-convex and decreasing in $\lambda_{i}$ 's. By the assumptions, $g(x)$ is decreasing in $x$, then the reverse hazard rate function of $X_{n: n}$ is decreasing in each $\lambda_{i}$.

Note that by the assumptions $x \tilde{r}(x)$ is convex in $x$. So, by Theorem $3.1 \mathrm{~b}$ (ii) (Theorem $3.2 \mathrm{a}$ (ii)) of [21], $\tilde{r}_{X_{n: n}}(x)$ is Schur convex. Thus, the result follows from Lemma 2.2.

In the following, we give some new results on the lifetimes of parallel systems in terms of the usual stochastic order.

Theorem 3.8. Let $X_{1}, \ldots, X_{n}\left(X_{1}^{*}, \ldots, X_{n}^{*}\right)$ be a set of independent nonnegative random variables with $X_{i} \sim \operatorname{ES}\left(\alpha, \lambda_{i}\right)\left(X_{i}^{*} \sim \operatorname{ES}\left(\alpha, \lambda_{i}^{*}\right)\right), i=1, \ldots, n$. If $F$ is DPRHR, then

$$
\left(\lambda_{1}, \ldots, \lambda_{n}\right) \stackrel{p}{\succeq}\left(\lambda_{1}^{*}, \ldots, \lambda_{n}^{*}\right) \Longrightarrow X_{n: n} \geq_{\mathrm{st}} X_{n: n}^{*} .
$$

Proof. The survival function of $X_{n: n}$ can be written as

$$
\bar{F}_{X_{n: n}}(t, \boldsymbol{a})=1-\prod_{i=1}^{n}\left(F\left(e^{a_{i}} t\right)\right)^{\alpha}
$$

where $a_{i}=\log \left(\lambda_{i}\right)$, for $i=1, \ldots, n$. Using Lemma 2.4 , it is enough to show that the function $\bar{F}_{X_{n: n}}(t, \boldsymbol{a})$ given in (3.19) is Schur-convex and decreasing in $a_{i}$ 's. To prove its Schur-convexity, it follows from Theorem 3.A.4. in [26] that we have to show that for $i \neq j$,

$$
\left(a_{i}-a_{j}\right)\left(\frac{\partial \bar{F}_{X_{n: n}}}{\partial a_{i}}-\frac{\partial \bar{F}_{X_{n: n}}}{\partial a_{j}}\right) \geq 0
$$

that is, for $i \neq j$,

$$
\alpha\left(a_{i}-a_{j}\right) \prod_{k=1}^{n}\left(F\left(e^{a_{k}} t\right)\right)^{\alpha}\left(t e^{a_{j}} \frac{f\left(e^{a_{j}} t\right)}{F\left(e^{a_{j}} t\right)}-t e^{a_{i}} \frac{f\left(e^{a_{i}} t\right)}{F\left(e^{a_{i}} t\right)}\right) \geq 0 .
$$

The assumption $x \tilde{r}(x)$ is decreasing in $x$ implies that the function $t e^{a_{i}} \tilde{r}\left(e^{a_{i}} t\right)$ is decreasing in $a_{i}$, for $i=1, \ldots, n$, from which it follows that (3.20) holds. The partial derivative of $\bar{F}_{X_{n: n}}(t, \boldsymbol{a})$ with respect to $a_{i}$ is negative, which in turn implies that the survival function of $X_{n: n}$ is decreasing in $a_{i}$ for $i=1, \ldots, n$. This completes the proof of the required result. 
In accordance with Theorem 3.8, for a parallel system with $n$ independent units following ES model, the DPRHR property of $F$ and the more heterogeneous in the $p$-largerer order the scale parameters is, the stochastically longer lifetime of the system and hence higher reliability will be achieved.

The above theorem immediately leads to the following corollary.

Corollary 3.5. Let $X_{1}, \ldots, X_{n}$ be a set of independent nonnegative random variables with $X_{i} \sim$ $\operatorname{ES}\left(\alpha, \lambda_{i}\right)\left(X_{i}^{*} \sim \operatorname{ES}\left(\alpha, \lambda_{i}^{*}\right)\right), i=1, \ldots, n$. Let $X_{1}^{*}, \ldots, X_{n}^{*}$ be i.i.d. random variables with common distribution. $F^{\alpha}(\tilde{\lambda} x)$, where $\tilde{\lambda}$ is the geometric mean of the $\lambda_{i}$ 's. If $F$ is DPRHR, then $X_{n: n} \geq_{\mathrm{st}} X_{n: n}^{*}$.

The above corollary gives a lower bound on the survival function of a parallel system with nonidentical components in terms of the one with i.i.d. components when the common scale parameter is the geometric mean of the scale parameters.

letting $\alpha=1$ in Theorem 3.8 leads to the following result proved by Khaledi et al. [11].

Corollary 3.6. Let $X_{1}, \ldots, X_{n}$ be a set of independent nonnegative random variables with $X_{i} \sim$ $G\left(\lambda_{i} x\right), i=1, \ldots, n$, where $G$ is an absolutely continuous distribution function with density function g. Let $X_{1}^{*}, \ldots, X_{n}^{*}$ be another set of independent nonnegative random variables with $X_{i}^{*} \sim G\left(\lambda_{i}^{*} x\right)$, $i=1, \ldots, n$. Let $\tilde{r}$ be the reverse hazard rate functions of $G$. If $x \tilde{r}(x)$ is decreasing in $x$, then

$$
\left(\lambda_{1}, \ldots, \lambda_{n}\right) \stackrel{p}{\succeq}\left(\lambda_{1}^{*}, \ldots, \lambda_{n}^{*}\right) \Longrightarrow X_{n: n} \geq_{\mathrm{st}} X_{n: n}^{*} .
$$

\section{Interdependent Variables with Archimedean Copulas}

Although in the literature, most researches on stochastic comparison on sample minimums assume the mutual independence among observations, recently some authors deal with the sample with dependent random variables. Here, we consider samples of ES random variables with a common Archimedean survival copula.

Theorem 4.1. Suppose for $i=1, \ldots, n, X_{i} \sim \operatorname{ES}\left(\alpha_{i}, \lambda\right)$ and $X_{i}^{*} \sim \operatorname{ES}\left(\alpha_{i}^{*}, \lambda\right)$ share a common Archimedean survival copula with generator $\phi$. Then, $X_{1: n} \leq_{\mathrm{st}} X_{1: n}^{*}$ if $\left(\alpha_{1}, \ldots, \alpha_{n}\right) \succeq^{\mathrm{W}}\left(\alpha_{1}^{*}, \ldots, \alpha_{n}^{*}\right)$.

Proof. The survival function of $X_{1: n}$ is given by

$$
\bar{F}_{X_{1: n}}(x)=p\left(X_{k}>x, 1 \leq k \leq n\right)=\phi\left(\sum_{i=1}^{n} \psi\left(1-(F(\lambda x))^{\alpha_{i}}\right)\right), \quad x>0 .
$$

Using Lemma 2.4, it is enough to show that the function $-\bar{F}_{X_{n: n}}(x)$ is Schur-convex and decreasing in $\alpha_{i}$ 's. The partial derivatives of $\bar{F}_{X_{1: n}}(x)$ with respect to $\alpha_{i}$ are

$$
\begin{gathered}
\frac{\partial \bar{F}_{X_{1: n}}(x)}{\partial \alpha_{i}}=-\frac{(F(\lambda x))^{\alpha_{i}} \log (F(\lambda x)) \phi^{\prime}\left(\sum_{i=1}^{n} \psi\left(1-(F(\lambda x))^{\alpha_{i}}\right)\right)}{\phi^{\prime}\left(\psi\left(1-(F(\lambda x))^{\alpha_{i}}\right)\right)} \geq 0, \\
\text { for all } \quad x>0 .
\end{gathered}
$$

Thus $\bar{F}_{X_{1: n}}(x)$ is increasing with respect to $\alpha_{i}$ 's. To prove its Schur-concavety, it follows from Theorem 3.A.4. in [26] that we have to show that for $i \neq j$,

$$
\left(\alpha_{i}-\alpha_{j}\right)\left(\frac{\partial \bar{F}_{X_{1: n}}(x)}{\partial \alpha_{i}}-\frac{\partial \bar{F}_{X_{1: n}}(x)}{\partial \alpha_{j}}\right) \leq 0,
$$


that is, for $i \neq j$,

$$
\begin{gathered}
-\log (F(\lambda x)) \phi^{\prime}\left(\sum_{i=1}^{n} \psi\left(1-(F(\lambda x))^{\alpha_{i}}\right)\right)\left(\alpha_{i}-\alpha_{j}\right) \\
\left(\frac{(F(\lambda x))^{\alpha_{i}}}{\phi^{\prime}\left(\psi\left(1-(F(\lambda x))^{\alpha_{i}}\right)\right)}-\frac{(F(\lambda x))^{\alpha_{j}}}{\phi^{\prime}\left(\psi\left(1-(F(\lambda x))^{\alpha_{j}}\right)\right)}\right) \leq 0 .
\end{gathered}
$$

Now, let us consider the function $g(\alpha)=\frac{(F(\lambda x))^{\alpha}}{\phi^{\prime}\left(\psi\left(1-(F(\lambda x))^{\alpha}\right)\right)}$. Taking derivative with respect to $\alpha$, we get

$$
\begin{aligned}
& g^{\prime}(\alpha) \stackrel{\operatorname{sgn}}{=}(F(\lambda x))^{\alpha} \log (F(\lambda x)) \phi^{\prime}\left(\psi\left(1-(F(\lambda x))^{\alpha}\right)\right) \\
& +\frac{(F(\lambda x))^{2 \alpha} \log (F(\lambda x))}{\phi^{\prime}\left(\psi\left(1-(F(\lambda x))^{\alpha}\right)\right)} \phi^{\prime \prime}\left(\psi\left(1-(F(\lambda x))^{\alpha}\right)\right) \geq 0 .
\end{aligned}
$$

Thus, $g(\alpha)$ is increasing with respect to $\alpha$ from which it follows that (4.2) holds. This completes the proof of the required result.

Theorem 4.2. Suppose, for $\boldsymbol{X} \sim \operatorname{ES}\left(\alpha, \lambda, \phi_{1}\right)$ and $\boldsymbol{X}^{*} \sim \operatorname{ES}\left(\alpha, \lambda^{*}, \phi_{2}\right), \phi_{1}$ or $\phi_{2}$ is log-convex, and $\psi_{2} \circ \phi_{1}$ is super-additive. Then, $X_{n: n} \geq_{\text {st }} X_{n: n}^{*}$ if $(i)\left(\lambda_{1}, \ldots, \lambda_{n}\right) \stackrel{p}{\succeq}\left(\lambda_{1}^{*}, \ldots, \lambda_{n}^{*}\right)$ and $F$ is DPRHR, or (ii) $\left(\lambda_{1}, \ldots, \lambda_{n}\right) \stackrel{\mathrm{w}}{\succeq}\left(\lambda_{1}^{*}, \ldots, \lambda_{n}^{*}\right)$ and $F$ is DRHR.

Proof. $X_{n: n}$ and $X_{n: n}^{*}$ have their respective distribution functions, for $x \geq 0$,

$$
\begin{gathered}
F_{X_{n: n}}(x)=\phi_{1}\left(\sum_{i=1}^{n} \psi_{1}\left(\left(F\left(\lambda_{i} x\right)\right)^{\alpha}\right)\right)=J\left(\boldsymbol{\lambda}, \alpha, x, \phi_{1}\right), \\
F_{X_{n: n}^{*}}(x)=\phi_{2}\left(\sum_{i=1}^{n} \psi_{2}\left(\left(F\left(\lambda_{i}^{*} x\right)\right)^{\alpha}\right)\right)=J\left(\boldsymbol{\lambda}^{*}, \alpha, x, \phi_{2}\right) .
\end{gathered}
$$

We only prove the case that $\phi_{1}$ is log-convex, and the other case can be finished similarly.

(i) Since $\phi_{1}$ is decreasing, we have

$$
\begin{gathered}
\frac{\partial J\left(\boldsymbol{\lambda}, \alpha, x, \phi_{1}\right)}{\partial \log \left(\lambda_{i}\right)}=\alpha x \lambda_{i} \tilde{r}\left(\lambda_{i} x\right)\left(F\left(\lambda_{i} x\right)\right)^{\alpha} \frac{\phi_{1}^{\prime}\left(\sum_{i=1}^{n} \psi_{1}\left(\left(F\left(\lambda_{i} x\right)\right)^{\alpha}\right)\right)}{\phi_{1}^{\prime}\left(\psi_{1}\left(\left(F\left(\lambda_{i} x\right)\right)^{\alpha}\right)\right)} \geq 0, \\
\text { for all } x>0,
\end{gathered}
$$

That is, $-J\left(\boldsymbol{\lambda}, \alpha, x, \phi_{1}\right)$ is decreasing in $\log \left(\lambda_{i}\right)$ for $i=1, \ldots, n$. Furthermore, for $i \neq j$,

$$
\begin{gathered}
\frac{\partial J\left(\boldsymbol{\lambda}, \alpha, x, \phi_{1}\right)}{\partial \log \left(\lambda_{i}\right)}-\frac{\partial J\left(\boldsymbol{\lambda}, \alpha, x, \phi_{1}\right)}{\partial \log \left(\lambda_{j}\right)}= \\
\alpha \phi_{1}^{\prime}\left(\sum_{i=1}^{n} \psi_{1}\left(\left(F\left(\lambda_{i} x\right)\right)^{\alpha}\right)\right)
\end{gathered}
$$




$$
\left(x \lambda_{i} \tilde{r}\left(\lambda_{i} x\right) \frac{\phi_{1}\left(\psi_{1}\left(\left(F\left(\lambda_{i} x\right)\right)^{\alpha}\right)\right)}{\phi_{1}^{\prime}\left(\psi_{1}\left(\left(F\left(\lambda_{i} x\right)\right)^{\alpha}\right)\right)}-x \lambda_{j} \tilde{r}\left(\lambda_{j} x\right) \frac{\phi_{1}\left(\psi_{1}\left(\left(F\left(\lambda_{j} x\right)\right)^{\alpha}\right)\right)}{\phi_{1}^{\prime}\left(\psi_{1}\left(\left(F\left(\lambda_{j} x\right)\right)^{\alpha}\right)\right)}\right) .
$$

Note that the log-convexity of $\phi_{1}$ implies the decreasing property of $\frac{\phi_{1}}{\phi_{1}^{\prime}}$. Since $\psi_{1}\left((F(\lambda x))^{\alpha}\right)$ is decreasing in $\lambda>0$, then $\frac{\phi_{1}\left(\psi_{1}\left((F(\lambda x))^{\alpha}\right)\right)}{\phi_{1}^{\prime}\left(\psi_{1}\left((F(\lambda x))^{\alpha}\right)\right)}$ is increasing in $\lambda>0$. Also the DPRHR property of $F$ implies that $x \lambda \tilde{r}(\lambda x)$ is decreasing in $\lambda>0$, and thus $x \lambda \tilde{r}(\lambda x) \frac{\phi_{1}\left(\psi_{1}\left((F(\lambda x))^{\alpha}\right)\right)}{\phi_{1}^{\prime}\left(\psi_{1}\left((F(\lambda x))^{\alpha}\right)\right)}$ is increasing in $\lambda>0$. So, for $i \neq j$,

$$
\left(\lambda_{i}-\lambda_{j}\right)\left(\frac{\partial J\left(\boldsymbol{\lambda}, \alpha, x, \phi_{1}\right)}{\partial \log \left(\lambda_{i}\right)}-\frac{\partial J\left(\boldsymbol{\lambda}, \alpha, x, \phi_{1}\right)}{\partial \log \left(\lambda_{j}\right)}\right) \leq 0 .
$$

Then Schur-convexity of $-J\left(\boldsymbol{\lambda}, \alpha, x, \phi_{1}\right)$ follows from Theorem 3.A.4. in [26]. Here it should be noted that, for two vectors $\boldsymbol{\lambda}$ and $\boldsymbol{\lambda}^{*}$, we have $\left(\lambda_{1}, \ldots, \lambda_{n}\right) \stackrel{p}{\succeq}\left(\lambda_{1}^{*}, \ldots, \lambda_{n}^{*}\right) \Longleftrightarrow$ $\left(\log \left(\lambda_{1}\right), \ldots, \log \left(\lambda_{n}\right)\right) \stackrel{\mathrm{w}}{\succeq}\left(\log \left(\lambda_{1}^{*}\right), \ldots, \log \left(\lambda_{n}^{*}\right)\right)$. According to Lemma $2.2\left(\lambda_{1}, \ldots, \lambda_{n}\right) \stackrel{p}{\succeq}$ $\left(\lambda_{1}^{*}, \ldots, \lambda_{n}^{*}\right)$ implies $-J\left(\lambda, \alpha, x, \phi_{1}\right) \geq-J\left(\lambda^{*}, \alpha, x, \phi_{1}\right)$. On the other hand, since $\psi_{2} \circ \phi_{1}$ is super-additive by Lemma 2.5 , we have $J\left(\lambda^{*}, \alpha, x, \phi_{1}\right) \leq J\left(\lambda^{*}, \alpha, x, \phi_{2}\right)$. So, it holds that

$$
J\left(\boldsymbol{\lambda}, \alpha, x, \phi_{1}\right) \leq J\left(\lambda^{*}, \alpha, x, \phi_{1}\right) \leq J\left(\lambda^{*}, \alpha, x, \phi_{2}\right) .
$$

That is, $X_{n: n} \geq_{\mathrm{st}} X_{n: n}^{*}$.

(ii) We omit its proof due to the similarity to that of Part (i).

letting $\alpha=1$ in Theorem 4.2 leads to the following corollary for scale model, verified by Li et al. [25].

Corollary 4.1. Suppose, for $\boldsymbol{X} \sim \mathrm{S}\left(\boldsymbol{\lambda}, \phi_{1}\right)$ and $\boldsymbol{X}^{*} \sim \mathrm{S}\left(\boldsymbol{\lambda}^{*}, \phi_{2}\right), \phi_{1}$ or $\phi_{2}$ is log-convex, and $\psi_{2} \circ \phi_{1}$ is super-additive. Then, $X_{n: n} \geq_{\mathrm{st}} X_{n: n}^{*}$ if $(i)\left(\lambda_{1}, \ldots, \lambda_{n}\right) \stackrel{p}{\succeq}\left(\lambda_{1}^{*}, \ldots, \lambda_{n}^{*}\right)$ and $F$ is DPRHR, or (ii) $\left(\lambda_{1}, \ldots, \lambda_{n}\right) \stackrel{\mathrm{w}}{\succeq}\left(\lambda_{1}^{*}, \ldots, \lambda_{n}^{*}\right)$ and $F$ is DRHR.

\section{Examples and Applications}

As the very popular fault tolerant structure, the $k$-out-of- $n$ system has been widely applied in industrial engineering and military systems. Particularly, the 1-out-of- $n$ system and the $n$-out-of- $n$ system correspond to the parallel system and series systems, respectively. In section 3, we carry out stochastic comparisons of lifetime of the parallel system and series systems arising from independent ES components in the sense of various stochastic orderings including the usual stochastic, reversed hazard rate and likelihood ratio orderings. In the following subsections, we present some distributions for which our results are applicable.

\subsection{Generalized exponential distribution}

To fit a model to lifetime data sets, it is observed in the literature that the generalized exponential distribution can be used as an alternative to the gamma, Weibull, and log-normal distributions. So, it 
is of interest to consider this distribution as the lifetimes of components of reliability systems. The cumulative distribution function of the GE distribution is

$$
G(x)=\left(1-e^{-\lambda x}\right)^{\alpha}, \quad x>0, \alpha>0, \lambda>0 .
$$

We denote this distribution by $\operatorname{GE}(\alpha, \lambda)$. It follows the ES model by letting the underlying distribution in the ES family be $F(x)=1-e^{-x}, x \geq 0$. Clearly, $F$ is IHR and IRHR. So, the condition of Theorem 3.1-3.4 holds. So the result of this Theorems simply yield results for series and parallel systems with independent heterogeneous generalized exponential components. As a consequence, this results is a generalization of Corresponding result due to [1].

The following corollary, verified by Balakrishnan et al. [1], follows immediately from Theorem 3.5.

Corollary 5.1. Let $X_{1}, \ldots, X_{n}$ be independent random variables with $X_{i} \sim \operatorname{GE}\left(\alpha_{i}, \lambda\right)$ and $X_{1}^{*}, \ldots, X_{n}^{*}$ be another set of independent random variables with $X_{i}^{*} \sim \operatorname{GE}\left(\alpha_{i}^{*}, \lambda\right), i=1, \ldots, n$. If $\sum_{i=1}^{n} \alpha_{i} \geq$ $\sum_{i=1}^{n} \alpha_{i}^{*}$, then for any $\lambda>0$, we have $X_{n: n} \geq_{\operatorname{lr}} X_{n: n}^{*}$.

From Theorem 3.6 and the fact that $F$ is DPRHR and $x^{2} \tilde{r}^{\prime}(x)$ is increasing in $x$, see lemma 2.1 of [12], we readily obtain the following corollary that generalizes the corresponding result in Theorem 10 (ii) of [1]. In particular the majorization assumption is relaxed to the super-majorization.

Corollary 5.2. Let $X_{1}, \ldots, X_{n}$ be independent random variables with $X_{i} \sim \operatorname{GE}\left(\alpha, \lambda_{i}\right)$ and $X_{1}^{*}, \ldots, X_{n}^{*}$ be another set of independent random variables with $X_{i}^{*} \sim \operatorname{GE}\left(\alpha, \lambda_{i}^{*}\right), i=1, \ldots, n$. Then for any $\alpha>0$,

$$
\left(\lambda_{1}, \ldots, \lambda_{n}\right) \stackrel{\mathrm{w}}{\succeq}\left(\lambda_{1}^{*}, \ldots, \lambda_{n}^{*}\right) \Longrightarrow X_{n: n} \geq_{\mathrm{rh}} X_{n: n}^{*}
$$

The following corollary, verified by Kundu et al. [21], follows immediately from Theorem 3.7.

Corollary 5.3. For $i=1, \ldots, n$, let $X_{i}$ and $X_{i}^{*}$ be two sets of mutually independent random variables with $X_{i} \sim \mathrm{GE}\left(\alpha_{i}, \lambda_{i}\right)$ and $X_{i}^{*} \sim \mathrm{GE}\left(\alpha_{i}, \lambda_{i}^{*}\right)$. Further, suppose that $\left\{\boldsymbol{\alpha} \in \mathbb{E}_{+}, \lambda, \lambda^{*} \in \mathbb{D}_{+}\right\}(\{\boldsymbol{\alpha} \in$ $\left.\left.\mathbb{D}_{+}, \boldsymbol{\lambda}, \boldsymbol{\lambda}^{*} \in \mathbb{E}_{+}\right\}\right)$. Then,

$$
\left(\lambda_{1}, \ldots, \lambda_{n}\right) \stackrel{\mathrm{w}}{\succeq}\left(\lambda_{1}^{*}, \ldots, \lambda_{n}^{*}\right) \Longrightarrow X_{n: n} \geq_{\mathrm{rh}} X_{n: n}^{*}
$$

Theorem 3.8 can be applied to the GE distribution, as proved in theorem 10 (i) of [1].

Remark 5.1. Khaledi and Kochar [12], proved special case of Theorem 3.8 when the baseline distribution in the ES model is exponential and $\alpha=1$.

\subsection{Generalized gamma distribution}

Recall that a random variable $X$ has a generalized gamma distribution, denoted by $X \sim \operatorname{GG}(p, q)$, when its density function has the following form

$$
g(x)=\frac{p}{\Gamma\left(\frac{q}{p}\right)} x^{q-1} e^{-x^{p}}, \quad x>0,
$$

where $p, q>0$ are the shapes parameters. The importance of this distribution lies in its flexibility in describing lifetime distributions ensuring their applications in survival analysis and reliability 
theory. It includes many important distributions like exponential $(p=q=1)$, Weibull $(p=q)$ and gamma $(p=1)$ as special cases. It is IHR when $p \geq 1, q \geq 1$. [11] proved that for $X \sim \operatorname{GG}(p, q)$, $x \tilde{r}(x)$ is a decreasing function of $x$ and $x^{2} \tilde{r}^{\prime}(x)$ is an increasing function of $x$ when $p<1$. So we apply the result of this paper when $F$ is a generalized gamma distribution which includes Weibull, gamma and exponential random variables as special cases. The result derived here strengthens and generalizes some of the results known in the literature.

\subsection{Other distributions}

A random variable $X$ is said to have power-generalized Weibull distribution, if its survival function is

$$
\bar{F}(x)=e^{1-\left(1+t^{v}\right)^{1 / \gamma}}, \quad t, v, \gamma>0 .
$$

It is DRHR when $v \leq \gamma, v \leq 1$, IHR when $v \geq \gamma, v \geq 1$. So, the condition of Theorem 3.1-3.4 holds. As [11] have shown, the power-generalized Weibull distribution satisfy the conditions of Theorem 3.8. Most of the other parametric families of practical importance have a scale parameter, such as Burr and half-normal distribution and the conditions of this paper can be easily checked for them.

\subsection{Auction theory}

In practice, it is of great interest for auctioneers to have a good understanding on the impact of the dependence among the bids. Our theoretical results in Section 4 throw some new light into this concern. For bids following ES model and coupled by Archimedean copulas, Theorem 4.2 tells that the final price in the FPA with less homogeneous and less positive dependent bids will be stochastically larger. From another perspective, this suggests the auctioneer be very cautious in releasing information because the dependence and homogeneity on bids strongly associate with the information and may harm the final price.

\section{Conclusions}

In this paper, for the first time, we have considered series and parallel systems with independent heterogeneous exponentiated scale components. These comparisons are made with respect to usual stochastic, reversed hazard rate and likelihood ratio orderings. We apply these results when $F$ is a generalized exponential, power-generalized Weibull and generalized gamma distribution which includes Weibull, gamma and exponential random variables as special cases. We also conducted stochastic comparison on the smallest (largest) order statistics from ES samples with Archimedean survival (Archimedean) copulas. The results of the paper extend some known results in the literature pertaining to the scale and exponentiated family of distributions.

\section{Acknowledgements}

The authors would like to thank the anonymous referees for valuable comments and suggestions. This work was supported by the Research Council of Yazd University.

\section{References}

[1] N. Balakrishnan, A. Haidari and K. Masoumifard, Stochastic comparisons of series and parallel systems with generalized exponential components, IEEE Trans. Reliab. 64 (2015), 333-348. 
[2] R. E. Barlow and F. Proschan, Statistical theory of reliability and life testing: Probability models (To Begin With, Silver Spring, MD. 1981)

[3] N. Balakrishnan and P. Zhao, Ordering properties of order statistics from heterogeneous populations: a review with an emphasis on some recent developments, Probab. Engrg. Inform. Sci. 27 (2013) $403-443$.

[4] J. L. Bon and E. Păltănea, Ordering properties of convolutions of exponential random variables, Lifetime Data Anal. 5 (1999) 185-192.

[5] H. A. David and H. N. Nagaraja, Order Statistics (Wiley, Hoboken, 2003).

[6] S. C. Dykstra, S. C. Kochar and J. Rojo, Stochastic comparisons of parallel systems of heterogeneous exponential components, J. Stat. Plan. Inference 65 (1997) 203-211.

[7] P. Embrechts, C. Kluppelberg and T. Mikosch, Modelling Extremal Events for Insurance and Finance (Springer, Berlin, 1997).

[8] R. Fang and X. Li, Advertising a second-price auction, J. Math. Econ. 61 (2015) 246-252.

[9] L. Fang and X. Zhang, Stochastic comparisons of parallel systems with exponentiated weibull components, Statist. Probab. Lett. 97 (2015) 25-31.

[10] T. Hu, Monotone coupling and stochastic ordering of order statistics, Syst. Sci. Math. Sci. 8 (1995) 209-214.

[11] B. E. Khaledi, S. Farsinezhad and S. C. Kochar, Stochastic comparisons of order statistics in the scale models, J. Statist. Plann. Inference 141 (2011) 276-286.

[12] B. E. Khaledi and S. C. Kochar, Some new results on stochastic comparisons results, J. Appl. Probab. 37 (2000) 1123-1128.

[13] B. E. Khaledi and S. C. Kochar, Dispersive ordering among linear combinations of uniform random variables, J. Statist. Plan. Inference 100 (2002) 13-21.

[14] B. E. Khaledi and S. C. Kochar, Weibull distribution: some stochastic comparisons results, J. Statist. Plann. Inference 136 (2006) 3121-3129.

[15] S. C. Kochar, Stochastic comparisons of order statistics and spacings: a review. ISRN Probab. Statist. (2012).

[16] S. C. Kochar and N. Torrado, On stochastic comparisons of largest order statistics in the scale model, Comm. Statist. Theory and Methods 44(19) (2015) 4132-4143.

[17] S. C. Kochar and M. Xu, Comparisons of parallel systems according to the convex transform order, $J$. Appl. Probab. 46 (2009) 342-352.

[18] S. C. Kochar and M. Xu, On the right spread order of convolutions of heterogeneous exponential random variables, J. Multivariate Anal. 101(1) (2010) 165-179.

[19] V. Krishna, Auction Theory (Academic Press, San Diego, 2010).

[20] A. Kundu and S. Chowdhury, Ordering properties of order statistics from heterogeneous exponentiated weibull models, Statist. Probab. Lett. 114 (2016) 119-127.

[21] A. Kundu, S. Chowdhury, A. K. Nanda and N. K. Hazra, Some results on majorization and their applications, J. Comput. Appl. Math. 301 (2016) 161-177.

[22] H. Li and X. Li, Stochastic Orders in Reliability and Risk (Springer, New York, 2013).

[23] X. Li, A note on expected rent in auction theory, Oper. Res. Lett. 33 (2005) 531-534.

[24] X. Li and R. Fang, Ordering properties of order statistics from random variables of Archimedean copulas with applications, J. Multivariate Anal. 133 (2015) 304-320.

[25] C. Li, R. Fang and X. Li, Stochastic somparisons of order statistics from scaled and interdependent random variables, Metrika (2015) 1-26.

[26] A. W. Marshall, I. Olkin and B. C. Arnold, Inequalities: Theory of Majorization and its Applications (Springer, New York, 2011).

[27] A. J. McNeil and J. Nešlehová, . Multivariate Archimedean Copulas, d-Monotone Functions and $\ell_{1}$ Norm Symmetric Distributions, Ann. Statist. (2009) 3059-3097.

[28] F. M. Menezes and P. K. Monteiro, An Introduction to Auction Theory (Oxford University Press, New York, 2005).

[29] P. Milgrom, Putting Auction Theory to Work (Cambridge University Press, New York, 2004).

[30] G. S. Mudholkar and D. K. Srivastava, Exponentiated weibull family for analyzing bathtub failure rate data, IEEE Trans. Rel. 42 (1993) 299-302. 
[31] R. B. Nelsen, An Introduction to Copulas (Springer, New York, 2006).

[32] G. Pledger and F. Proschan, Comparisons of order statistics and of spacings from heterogeneous distributions. In: Rustagi, J.S. (Ed.), Optimizing Methods in Statistics. Academic Press, New York, (1971) 89-113.

[33] F. Proschan and J. Sethuraman, Stochastic comparisons of order statistics from heterogeneous populations, with applications in reliability, J. Multivariate Anal. 6 (1976) 608-616.

[34] M. Shaked and J. G. Shanthikumar, Stochastic Orders (Springer, New York, 2007).

[35] N. Torrado, On magnitude orderings between smallest order statistics from heterogeneous beta distributions, J. Math. Anal. Appl. 426(2) (2015) 824-838.

[36] P. Zhao, L. Wang and Y. Zhang, On extreme order statistics from heterogeneous beta distributions with applications, Comm. Statist. Theory and Methods just-accepted (2016). 